\title{
Entropy Dissipation Rate and Convergence in Kinetic Equations
}

\author{
L. Desvillettes
}

Centre de Mathématiques Appliquées, Ecole Normale Supérieure, 45 Rue d'Ulm, F-75230 Paris Cedex 05, France

\begin{abstract}
We give a lower bound of the entropy dissipation rate of Kač, Boltzmann and Fokker-Planck-Landau equations. We apply this estimate to the problem of the speed of convergence to equilibrium in large time for the Boltzmann equation.
\end{abstract}

\section{Introduction}

Rarefied gas dynamics is usually described by the Boltzmann equation

$$
\partial_{t} f+v \cdot \nabla_{x} f=Q(f, f),
$$

where $f(t, x, v)$ is the density of particles which at time $t$ and point $x$, move with velocity $v$, and $Q$ is a quadratic collision term described in [3, 4 and 12].

A simpler monodimensional model has been introduced by Kač in [9]:

$$
\partial_{t} f+v \partial_{x} f=\widetilde{Q}(f, f)
$$

where $\widetilde{Q}$ is defined in [9] or [11].

The asymptotics of the Boltzmann equation when the grazing collisions become predominant formally leads to the Fokker-Planck-Landau equation

$$
\partial_{t} f+v \cdot \nabla_{x} f=Q^{\prime}(f, f)
$$

where $Q^{\prime}$ is still a quadratic collision term. The formal derivation of this equation and the form of $Q^{\prime}$ may be found in [4] or [10].

According to Boltzmann's $H$-theorem, the entropy dissipation rate

$$
\int_{v \in \mathbb{R}^{3}} Q(f, f)(v) \log f(v) d v
$$

is nonpositive. Moreover, it is equal to 0 if and only if $f$ is a Maxwellian (cf. [12]). The same property holds for the collision term $Q^{\prime}$, and for $\widetilde{Q}$ with the additional prescription that the Maxwellian is of bulk velocity 0 . 
Therefore, in order to get a better understanding of the phenomena appearing when the entropy dissipation term tends to 0 , it is useful to obtain a lower bound of it in terms of some distance from $f$ to the space of Maxwellians.

Such an estimate measures the speed of convergence to equilibrium when the entropy dissipation tends to 0 . This situation occurs for example when the time $t$ tends to infinity in Eq. (1), (2) and (3), or when the mean free path $\varepsilon$ tends to zero in the equation

$$
\partial_{t} f+v \cdot \nabla_{x} f=\frac{1}{\varepsilon} Q(f, f) .
$$

Accordingly, in Sect. 2, we give a lower bound of the entropy dissipation term for the Kač equation in terms of a distance to the Maxwellian states of the type

$$
\inf _{m \in \tilde{\Gamma}} \int|\log f(v)-m(v)| d v
$$

where $\tilde{\Gamma}$ is the space of logarithms of Maxwellians with zero bulk velocity.

We extend this result in Sect. 3 to the case of the Boltzmann collision kernel $Q$, and in Sect. 4 to the case of the Fokker-Planck-Landau collision kernel $Q^{\prime}$.

We explain in Sect. 5 how these results give classical estimates when applied to the linearised kernels of $\widetilde{Q}, Q$ and $Q^{\prime}$.

In Sect. 6, we apply the previous estimates to investigate the long time behaviour of the Boltzmann equation.

\section{On the Kač Collision Kernel}

The Kač equation,

$$
\partial_{t} f+v \partial_{x} f=\widetilde{Q}(f, f)
$$

models a one-dimensional gas in which all collisions conserving the energy are equiprobable (cf. [9] or [11]). Therefore, its collision kernel $\widetilde{Q}$ is the following:

$$
\widetilde{Q}(f, f)(v)=\int_{v_{1} \in \mathbb{R}} \int_{\theta=0}^{2 \pi}\left\{f\left(v^{\prime}\right) f\left(v_{1}^{\prime}\right)-f(v) f\left(v_{1}\right)\right\} \frac{d \theta}{2 \pi} d v_{1},
$$

where

$$
\begin{aligned}
& v^{\prime}=v \cos \theta+v_{1} \sin \theta, \\
& v_{1}^{\prime}=-v \sin \theta+v_{1} \cos \theta .
\end{aligned}
$$

The theorem below is a partial answer to a question of McKean (cf. [11], p. $365 ; 13 \mathrm{~b}])$.

We shall denote by $U$ the set of the convex, continuous and even function from $\mathbb{R}$ to $\mathbb{R}$ such that for all $x$ in $\mathbb{R}$,

$$
0 \leqq \phi(x) \leqq x\left(e^{x}-1\right)
$$

Moreover, if $B$ is a function from $\mathbb{R}_{+}^{*}$ to $\mathbb{R}_{+}^{*}$, we introduce the set

$$
L_{B}^{p}=\left\{f \in L^{p} / \text { for all } v \text { such that }|v| \leqq R, f(v) \geqq B_{R}\right\},
$$


and $\tilde{\Gamma}$ is the space of logarithms of Maxwellians with zero bulk velocity

$$
\tilde{\Gamma}=\left\{a+b v^{2} / a, b \in \mathbb{R}\right\} .
$$

Theorem 1. Let $\widetilde{Q}$ be the Kač collision kernel, and $R$ be strictly positive. Then there exists a strictly positive $K_{R}$ such that for all $f$ in $L_{B}^{1}$ and all $\phi$ in $U$,

$$
-\int_{v \in \mathbb{R}} \widetilde{Q}(f, f)(v) \log f(v) d v \geqq \frac{1}{4} B_{R}^{2} \pi R^{2} \phi\left(\frac{K_{R}}{\pi R^{2}} \inf _{m \in \tilde{\Gamma}} \int_{|v| \leqq R}|\log f(v)-m(v)| d v\right) .
$$

Proof of Theorem 1. Boltzmann's $H$-theorem ensures that

$$
\begin{aligned}
&- \int_{v \in \mathbb{R}} \widetilde{Q}(f, f)(v) \log f(v) d v \\
&= \frac{1}{4} \int_{v \in \mathbb{R}} \int_{v_{1} \in \mathbb{R}} \int_{\theta=0}^{2 \pi}\left\{f\left(v^{\prime}\right) f\left(v_{1}^{\prime}\right)-f(v) f\left(v_{1}\right)\right\}\left\{\log \left(f\left(v^{\prime}\right) f\left(v_{1}^{\prime}\right)\right)-\log \left(f(v) f\left(v_{1}\right)\right)\right\} \\
& \cdot \frac{d \theta}{2 \pi} d v_{1} d v \\
& \geqq \frac{1}{4} B_{R}^{2} \int_{v^{2}+v_{1}^{2} \leqq R^{2}} \int_{\theta=0}^{2 \pi} \lambda\left(\log f\left(v^{\prime}\right)+\log f\left(v_{1}^{\prime}\right)-\log f(v)-\log f\left(v_{1}\right)\right) \frac{d \theta}{2 \pi} d v_{1} d v,
\end{aligned}
$$

with

Therefore

$$
\lambda(x)=x\left(e^{x}-1\right)
$$

$$
\begin{aligned}
& -\int_{v \in \mathbb{R}} \tilde{Q}(f, f)(v) \log f(v) d v \\
& \geqq \frac{1}{4} B_{R}^{2} \pi R^{2} \phi\left(\frac{1}{\pi R^{2}} \int_{v^{2}+v_{1}^{2} \leqq R^{2}} \int_{\theta=0}^{2 \pi}\left|\log f\left(v^{\prime}\right)+\log f\left(v_{1}^{\prime}\right)-\log f(v)-\log f\left(v_{1}\right)\right|\right. \\
& \left.\quad \cdot \frac{d \theta}{2 \pi} d v_{1} d v\right)
\end{aligned}
$$

by Jensen's inequality.

Before going further in the proof, we need the following lemmas:

\section{Lemma 1.}

$$
\begin{gathered}
\int_{v^{2}+v_{1}^{2} \leqq R^{2}} \int_{\theta=0}^{2 \pi}\left|\log f\left(v^{\prime}\right)+\log f\left(v_{1}^{\prime}\right)-\log f(v)-\log f\left(v_{1}\right)\right| \frac{d \theta}{2 \pi} d v_{1} d v \\
\geqq \inf _{\text {functions } T} \int_{v^{2}+v_{1}^{2} \leqq R^{2}}\left|\log f(v)+\log f\left(v_{1}\right)-T\left(v^{2}+v_{1}^{2}\right)\right| d v_{1} d v .
\end{gathered}
$$

Proof of Lemma 1. Let us introduce the following notations:

$$
\begin{gathered}
g\left(v, v_{1}\right)=\log f(v)+\log f\left(v_{1}\right), \\
R_{\psi}=\text { rotation of angle } \psi,
\end{gathered}
$$


and compute

$$
\begin{gathered}
\int_{\theta=0}^{2 \pi}\left|\log f\left(v^{\prime}\right)+\log f\left(v_{1}^{\prime}\right)-\log f(v)-\log f\left(v_{1}\right)\right| \frac{d \theta}{2 \pi}=\int_{\theta=0}^{2 \pi}\left|g\left(R_{\theta}\left(v, v_{1}\right)\right)-g\left(v, v_{1}\right)\right| \frac{d \theta}{2 \pi} \\
\geqq\left|\int_{\theta=0}^{2 \pi} g\left(R_{\theta}\left(v, v_{1}\right)\right) \frac{d \theta}{2 \pi}-g\left(v, v_{1}\right)\right| .
\end{gathered}
$$

But

$$
\int_{\theta=0}^{2 \pi} g\left(R_{\theta}\left(v, v_{1}\right)\right) \frac{d \theta}{2 \pi}
$$

is a function depending only on $v^{2}+v_{1}^{2}$, which concludes the proof.

Lemma 2. For all strictly positive $R$, there exists a strictly positive $K_{R}$ such that

$$
\begin{aligned}
& \inf _{\text {functions } T} \int_{v^{2}+v_{1}^{2} \leqq R^{2}}\left|\log f(v)+\log f\left(v_{1}\right)-T\left(v^{2}+v_{1}^{2}\right)\right| d v_{1} d v \\
& \geqq K_{R} \inf _{m \in \tilde{\Gamma}} \int_{|v| \leqq R}|\log f(v)-m(v)| d v .
\end{aligned}
$$

Proof of Lemma 2. Let $\tilde{M}$ be the space of functions depending only on $v^{2}+v_{1}^{2}$, and $\tilde{L}$ be the following operator:

$$
\widetilde{L}: t \in L^{1}(|v| \leqq R) / \widetilde{\Gamma} \rightarrow \tilde{L} t\left(v, v_{1}\right)=t(v)+t\left(v_{1}\right) \in L^{1}\left(v^{2}+v_{1}^{2} \leqq R^{2}\right) / \tilde{M} .
$$

The operator $\tilde{L}$ is clearly linear and one-one (cf. [3] or [12] in the more complicated case of the Boltzmann collision kernel).

Observe that for all $t$ in $L^{1}(|v| \leqq R)$,

$$
\begin{aligned}
& \inf _{\text {functions } T} \int_{v^{2}+v_{1}^{2} \leqq R^{2}}\left|t(v)+t\left(v_{1}\right)-T\left(v^{2}+v_{1}^{2}\right)\right| d v_{1} d v \\
& \leqq \inf _{a, b \in \mathbb{R}} \int_{v^{2}+v_{1}^{2} \leqq R^{2}}\left|t(v)+t\left(v_{1}\right)-a\left(v^{2}+v_{1}^{2}\right)-2 b\right| d v_{1} d v \\
& \leqq 4 R \inf _{m \in \tilde{\Gamma}} \int_{|v| \leqq R}|t(v)-m(v)| d v .
\end{aligned}
$$

Therefore, the operator $\tilde{L}$ is continuous.

In order to apply the open mapping theorem, we have to prove that the image of $\tilde{L}$ is closed. Assume that there exists a sequence $t_{n}$ in $L^{1}(|v| \leqq R) / \widetilde{\Gamma}$ and $t$ in $L^{1}\left(v^{2}+v_{1}^{2} \leqq R^{2}\right) / \tilde{M}$ such that $t_{n}(v)+t_{n}\left(v_{1}\right)$ tends to $t\left(v, v_{1}\right)$ in $L^{1}\left(v^{2}+v_{1}^{2} \leqq R^{2}\right) / \tilde{M}$. Then, there exist a sequence $k_{n}$ in $L^{1}(|v| \leqq R)$, a sequence $T_{n}$ depending only on $v^{2}+v_{1}^{2}$ and $g$ in $L^{1}\left(v^{2}+v_{1}^{2} \leqq R^{2}\right)$ such that $t_{n}$ is the natural projection of $k_{n}$ on $L^{1}(|v| \leqq R) / \tilde{\Gamma}, t$ is the natural projection of $g$ on $L^{1}\left(v^{2}+v_{1}^{2} \leqq R^{2}\right) / \tilde{M}$, and

$$
k_{n}(v)+k_{n}\left(v_{1}\right)+T_{n}\left(v^{2}+v_{1}^{2}\right) \rightarrow g\left(v, v_{1}\right)
$$

in $L^{1}\left(v^{2}+v_{1}^{2} \leqq R^{2}\right)$.

Next, we introduce the differential operator

$$
v_{1} \frac{\partial}{\partial v}-v \frac{\partial}{\partial v_{1}},
$$


which has the following property:

$$
\left(v_{1} \frac{\partial}{\partial v}-v \frac{\partial}{\partial v_{1}}\right) T\left(v^{2}+v_{1}^{2}\right)=0
$$

According to (7),

$$
\left\{v_{1} \frac{\partial}{\partial v}-v \frac{\partial}{\partial v_{1}}\right\}\left(k_{n}\left(v_{1}\right)+k_{n}(v)+T_{n}\left(v^{2}+v_{1}^{2}\right)\right)=v_{1} k_{n}^{\prime}(v)-v k_{n}^{\prime}\left(v_{1}\right)
$$

converges in $W^{-1,1}$. Taking the double partial derivative of this expression with respect to $v, v_{1}$, we obtain that

$$
k_{n}^{\prime \prime}(v)-k_{n}^{\prime \prime}\left(v_{1}\right)
$$

converges in $W^{-3,1}$. Therefore, there exists a sequence of real numbers $b_{n}$ such that

$$
k_{n}^{\prime \prime}(v)+b_{n}
$$

converges in $W^{-3,1}$. Then, there exists a sequence of real numbers $c_{n}$ such that

$$
k_{n}^{\prime}(v)+b_{n} v+c_{n}
$$

converges in $W^{-2,1}$ and, according to (8),

converges in $W^{-2,1}$.

$$
k_{n}^{\prime}(v)+b_{n} v
$$

But (8) ensures that

$$
\frac{\partial}{\partial v_{1}}\left\{v_{1} k_{n}^{\prime}(v)-v k_{n}^{\prime}\left(v_{1}\right)\right\}
$$

converges in $W^{-2,1}$, and therefore

$$
k_{n}^{\prime}(v)-v k_{n}^{\prime \prime}\left(v_{1}\right)
$$

converges in $W^{-2,1}$. Then, (9) together with (10) ensures that

$$
k_{n}^{\prime \prime}(v)+b_{n}
$$

converges in $W^{-2,1}$. According to (9), we obtain that

$$
k_{n}^{\prime}(v)+b_{n} v
$$

converges in $W^{-1,1}$, and therefore there exists a sequence of real numbers $a_{n}$ such that

$$
k_{n}(v)+\frac{1}{2} b_{n} v^{2}+a_{n}
$$

converges weakly in $L^{1}$.

Therefore, $t_{n}$ converges weakly in $L^{1}(|v| \leqq R) / \widetilde{\Gamma}$ and the image of $\tilde{L}$ is closed. Applying the theorem of the open mapping to $\widetilde{L}$, we obtain a strictly positive $K_{R}$ such that:

$$
\inf _{\text {functions } T} \int_{v^{2}+v_{1}^{2} \leqq R^{2}}\left|t(v)+t\left(v_{1}\right)-T\left(v^{2}+v_{1}^{2}\right)\right| d v_{1} d v \geqq K_{R} \inf _{m \in \tilde{\Gamma}} \int_{|v| \leqq R}|t(v)-m(v)| d v .
$$


Injecting $t=\log f$ in this estimate, we obtain Lemma 2.

The proof of Theorem 1 easily follows from Lemmas 1 and 2 together with estimate (5).

\section{On the Boltzmann Collision Kernel}

For the derivation of Boltzmann collision kernel, we refer to [3, 4 or 12]. We take

$$
Q(f, f)=\int_{v_{1} \in \mathbb{R}^{3}} \int_{\omega \in s^{2}}\left\{f\left(v^{\prime}\right) f\left(v_{1}^{\prime}\right)-f(v) f\left(v_{1}\right)\right\} B\left(v, v_{1}, \omega\right) d \omega d v_{1}
$$

with

$$
\begin{aligned}
& v^{\prime}=v+\omega \cdot\left(\left(v_{1}-v\right) \cdot \omega\right), \\
& v_{1}^{\prime}=v_{1}-\omega \cdot\left(\left(v_{1}-v\right) \cdot \omega\right),
\end{aligned}
$$

and $B$ is a nonnegative collision cross-section, depending on the collision process.

According to Boltzmann's $H$-theorem, the equilibrium is obtained for Maxwellian densities if $B$ is strictly positive a.e. In order to obtain an estimate of the type (4), we need a stronger assumption on $B$. From now on, we shall assume that for all strictly positive $R$, there exists a strictly positive $C_{R}$ such that

$$
B\left(v, v_{1}, \omega\right) \geqq C_{R}\left|\omega \cdot \frac{v_{1}-v}{\left|v_{1}-v\right|}\right|
$$

for all $v, v_{1}$ such that $v^{2}+v_{1}^{2} \leqq R^{2}$. Note that this assumption is satisfied in the classical cases of hard spheres and inverse power forces with or without angular cutoff assumption (cf. [3, 4, 8 and 12]).

We keep in this section the notations of Sect. 2. Moreover, we introduce $\Gamma$, the space of logarithms of Maxwellians:

$$
\Gamma=\left\{a v^{2}+b \cdot v+c / a, c \in \mathbb{R}, b \in \mathbb{R}^{3}\right\},
$$

we denote by $|A|$ the Lebesgue measure of the set $A$, and by $S^{N}$ the sphere of dimension $N$.

The main result of this section is the following:

Theorem 2. Let $Q$ be the Boltzmann collision kernel with $B$ as in (12) and $R$ be strictly positive. Then there exists a strictly positive $K_{R}$ such that, for all $f$ in $L_{B}^{1}$ and all $\phi$ in $U$,

$$
\begin{gathered}
-\int_{v \in \mathbb{R}^{3}} Q(f, f)(v) \log f(v) d v \geqq \frac{1}{4} B_{R}^{2}\left|S^{5}\right|\left|S^{2}\right| R^{6} C_{R} \phi \\
\cdot\left(\frac{K_{R}}{\left|S^{5}\right|\left|S^{2}\right| R^{6}} \inf _{m \in \Gamma} \int_{|v| \leqq R}|\log f(v)-m(v)| d v\right) .
\end{gathered}
$$


Proof of Theorem 2. By Boltzmann's $H$-theorem,

$$
\begin{aligned}
- & \int_{v \in \mathbb{R}^{3}} Q(f, f)(v) \log f(v) d v \\
= & \frac{1}{4} \int_{v \in \mathbb{R}^{3}} \int_{v_{1} \in \mathbb{R}^{3}} \int_{\omega \in S^{2}}\left\{f\left(v^{\prime}\right) f\left(v_{1}^{\prime}\right)-f(v) f\left(v_{1}\right)\right\} \\
& \cdot\left\{\log \left(f\left(v^{\prime}\right) f\left(v_{1}^{\prime}\right)\right)-\log \left(f(v) f\left(v_{1}\right)\right)\right\} B\left(v, v_{1}, \omega\right) d \omega d v_{1} d v \\
\geqq & \frac{1}{4} B_{R}^{2} C_{R} \int_{v^{2}+v_{1}^{2} \leqq R^{2}} \int_{\omega \in S^{2}} \lambda\left(\log f\left(v^{\prime}\right)+\log f\left(v_{1}^{\prime}\right)-\log f(v)-\log f\left(v_{1}\right)\right) \\
& \cdot\left|\omega \cdot \frac{v_{1}-v}{\left|v_{1}-v\right|}\right| d \omega d v_{1} d v
\end{aligned}
$$

with

$$
\lambda(x)=x\left(e^{x}-1\right)
$$

And therefore

$$
\begin{aligned}
& -\int_{v \in \mathbb{R}^{3}} Q(f, f)(v) \log f(v) d v \geqq \frac{1}{4} B_{R}^{2} C_{R}\left|S^{2}\right|\left|S^{5}\right| R^{6} \phi \\
& \cdot\left(\frac{1}{\left|S^{2}\right|\left|S^{5}\right| R^{6}} \int_{v^{2}+v_{1}^{2} \leqq R^{2}} \int_{\omega \in S^{2}}\left|\log f\left(v^{\prime}\right)+\log f\left(v_{1}^{\prime}\right)-\log f(v)-\log f\left(v_{1}\right)\right|\right. \\
& \left.\cdot\left|\omega \cdot \frac{v_{1}-v}{\left|v_{1}-v\right| \mid}\right| d \omega d v_{1} d v\right)
\end{aligned}
$$

by Jensen's inequality (because $\left.\int_{v^{2}+v_{1}^{2} \leqq R^{2}} \int_{\omega \in S^{2}}\left|\omega \cdot \frac{v_{1}-v}{\left|v_{1}-v\right|}\right| d \omega d v_{1} d v=\left|S^{2}\right|\left|S^{5}\right| R^{6}\right)$.

In the sequel, we need the following lemmas:

\section{Lemma 3.}

$$
\begin{aligned}
& \int_{v^{2}+v_{1}^{2} \leqq R^{2}} \int_{\omega \in S^{2}}\left|\log f\left(v^{\prime}\right)+\log f\left(v_{1}^{\prime}\right)-\log f(v)-\log f\left(v_{1}\right)\right|\left|\omega \cdot \frac{v_{1}-v}{\left|v_{1}-v\right|}\right| d \omega d v_{1} d v \\
& \geqq \inf _{\text {functions } T} \int_{v^{2}+v_{1}^{2} \leqq R^{2}}\left|\log f(v)+\log f\left(v_{1}\right)-T\left(v+v_{1}, v^{2}+v_{1}^{2}\right)\right| d v_{1} d v .
\end{aligned}
$$

Proof of Lemma 3. Let us introduce the notation: $g\left(v, v_{1}\right)=\log f(v)+\log f\left(v_{1}\right)$. We compute

$$
\begin{aligned}
& \int_{v^{2}+v_{1}^{2} \leqq R^{2}} \int_{\omega \in S^{2}}\left|\log f\left(v^{\prime}\right)+\log f\left(v_{1}^{\prime}\right)-\log f(v)-\log f\left(v_{1}\right)\right|\left|\omega \cdot \frac{v_{1}-v}{\left|v_{1}-v\right|}\right| d \omega d v_{1} d v \\
& =\int_{v^{2}+v_{1}^{2} \leqq R^{2}} \int_{\omega \in S^{2}}\left|g\left(v^{\prime}, v_{1}^{\prime}\right)-g\left(v, v_{1}\right)\right|\left|\omega \cdot \frac{v_{1}-v}{\left|v_{1}-v\right|}\right| d \omega d v_{1} d v .
\end{aligned}
$$

Then, we introduce the following change of variables:

$$
\sigma=S(\omega)
$$


with

$$
S(\omega)=2\left(\omega \cdot \frac{v_{1}-v}{\left|v_{1}-v\right|}\right) \cdot \omega-\frac{v_{1}-v}{\left|v_{1}-v\right|} .
$$

The Jacobian of $S$ is

and

$$
\left|\omega \cdot \frac{v_{1}-v}{\left|v_{1}-v\right|}\right|^{-1}
$$

with

$$
\left(v^{\prime}, v_{1}^{\prime}\right)=U_{\sigma}\left(v, v_{1}\right)
$$

$$
U_{\sigma}\left(v, v_{1}\right)=\frac{1}{2}\left(v+v_{1}+\left|v-v_{1}\right| \sigma, v+v_{1}-\left|v-v_{1}\right| \sigma\right) .
$$

According to (11), we obtain:

$$
\begin{aligned}
& \int_{v^{2}+v_{1}^{2} \leqq R^{2}} \int_{\omega \in S^{2}}\left|\log f\left(v^{\prime}\right)+\log f\left(v_{1}^{\prime}\right)-\log f(v)-\log f\left(v_{1}\right)\right|\left|\omega \cdot \frac{v_{1}-v}{\left|v_{1}-v\right|}\right| d \omega d v_{1} d v \\
& \geqq \int_{v^{2}+v_{1}^{2} \leqq R^{2}} \int_{\sigma \in S^{2}}\left|g\left(U_{\sigma}\left(v, v_{1}\right)\right)-g\left(v, v_{1}\right)\right| d \sigma d v_{1} d v \\
& \geqq \int_{v^{2}+v_{1}^{2} \leqq R^{2}}\left|\int_{\sigma \in S^{2}} g\left(U_{\sigma}\left(v, v_{1}\right)\right) d \sigma-g\left(v, v_{1}\right)\right| d v_{1} d v .
\end{aligned}
$$

But $U_{\sigma}\left(v, v_{1}\right)$ depends only on $\left(v+v_{1}, v^{2}+v_{1}^{2}\right)$, and therefore

$$
\begin{aligned}
& \int_{v^{2}+v_{1}^{2} \leqq R^{2}} \int_{\omega \in S^{2}}\left|\log f\left(v^{\prime}\right)+\log f\left(v_{1}^{\prime}\right)-\log f(v)-\log f\left(v_{1}\right)\right|\left|\omega \cdot \frac{v_{1}-v}{\left|v_{1}-v\right|}\right| d \omega d v_{1} d v \\
& \geqq \inf _{\text {functions } T} \int_{v^{2}+v_{1}^{2} \leqq R^{2}}\left|g\left(v, v_{1}\right)-T\left(v+v_{1}, v^{2}+v_{1}^{2}\right)\right| d v_{1} d v \\
& \geqq \inf _{\text {functions } T} \int_{v^{2}+v_{1}^{2} \leqq R^{2}}\left|\log f(v)+\log f\left(v_{1}\right)-T\left(v+v_{1}, v^{2}+v_{1}^{2}\right)\right| d v_{1} d v
\end{aligned}
$$

which concludes the proof of the lemma.

Lemma 4. For all strictly positive $R$, there exists a strictly positive $K_{R}$ such that

$$
\begin{aligned}
& \underset{\text { functions } T}{\inf } \int_{v^{2}+v_{1}^{2} \leqq R^{2}}\left|\log f(v)+\log f\left(v_{1}\right)-T\left(v+v_{1}, v^{2}+v_{1}^{2}\right)\right| d v_{1} d v \\
& \geqq K_{R} \inf _{m \in \Gamma} \int_{|v| \leqq R}|\log f(v)-m(v)| d v .
\end{aligned}
$$

Proof of Lemma 4. Let $M$ be the space of functions depending only on $\left(v+v_{1}\right.$, $\left.v^{2}+v_{1}^{2}\right)$, and let $L$ be the operator

$$
L: t \in L^{1}(|v| \leqq R) / \Gamma \rightarrow L t\left(v, v_{1}\right)=t(v)+t\left(v_{1}\right) \in L^{1}\left(v^{2}+v_{1}^{2} \leqq R^{2}\right) / M .
$$

The operator $L$ is clearly linear and one-one (cf. [3 or 12]). 
Observe that for all $t$ in $L^{1}(|v| \leqq R)$,

$$
\begin{aligned}
& \inf _{\text {functions } T} \int_{v^{2}+v_{1}^{2} \leqq R^{2}}\left|t(v)+t\left(v_{1}\right)-T\left(v^{2}+v_{1}^{2}\right)\right| d v_{1} d v \\
& \leqq \inf _{a, c \in \mathbb{R}, b \in \mathbb{R}^{3}} \int_{v^{2}+v_{1}^{2} \leqq R^{2}}\left|t(v)+t\left(v_{1}\right)-a\left(v^{2}+v_{1}^{2}\right)-b \cdot\left(v+v_{1}\right)-2 c\right| d v_{1} d v \\
& \leqq 16 R^{3} \inf _{m \in \Gamma} \int_{|v| \leqq R}|\log t(v)-m(v)| d v
\end{aligned}
$$

Therefore, the operator $L$ is continuous.

In order to apply the open mapping theorem, we have to prove that the image of $L$ is closed. Suppose that there exist a sequence $t_{n}$ in $L^{1}(|v| \leqq R) / \Gamma$ and $t$ in $L^{1}\left(v^{2}+v_{1}^{2} \leqq R^{2}\right) / M$ such that $t_{n}(v)+t_{n}\left(v_{1}\right)$ tends to $t\left(v, v_{1}\right)$ in $L^{1}\left(v^{2}+v_{1}^{2} \leqq R^{2}\right) / M$. Then, there exist a sequence $k_{n}$ in $L^{1}(|v| \leqq R)$, a sequence $T_{n}$ depending only on $v+v_{1}, v^{2}+v_{1}^{2}$ and $g$ in $L^{1}\left(v^{2}+v_{1}^{2} \leqq R^{2}\right)$ such that $t_{n}$ is the natural projection of $k_{n}$ on $L^{1}(|v|<R) / \Gamma, t$ is the natural projection of $g$ on $L^{1}\left(v^{2}+v_{1}^{2} \leqq R^{2}\right) / M$, and

$$
k_{n}(v)+k_{n}\left(v_{1}\right)+T_{n}\left(v^{2}+v_{1}^{2}\right) \rightarrow g\left(v, v_{1}\right)
$$

in $L^{1}\left(v^{2}+v_{1}^{2} \leqq R^{2}\right)$.

From now on, we shall write

$$
v=\left(x_{1}, x_{2}, x_{3}\right), \quad v_{1}=\left(y_{1}, y_{2}, y_{3}\right) .
$$

We introduce the following differential operator:

$$
\bar{\nabla}=\left(\begin{array}{l}
\left(y_{2}-x_{2}\right)\left(\frac{\partial}{\partial x_{1}}-\frac{\partial}{\partial y_{1}}\right)-\left(y_{1}-x_{1}\right)\left(\frac{\partial}{\partial x_{2}}-\frac{\partial}{\partial y_{2}}\right) \\
\left(y_{3}-x_{3}\right)\left(\frac{\partial}{\partial x_{2}}-\frac{\partial}{\partial y_{2}}\right)-\left(y_{2}-x_{2}\right)\left(\frac{\partial}{\partial x_{3}}-\frac{\partial}{\partial y_{3}}\right) \\
\left(y_{1}-x_{1}\right)\left(\frac{\partial}{\partial x_{3}}-\frac{\partial}{\partial y_{3}}\right)-\left(y_{3}-x_{3}\right)\left(\frac{\partial}{\partial x_{1}}-\frac{\partial}{\partial y_{1}}\right)
\end{array}\right)
$$

We observe that

accordingly,

$$
\bar{\nabla} T\left(v+v_{1}, v^{2}+v_{1}^{2}\right)=0
$$

converges in $W^{-1,1}$. Therefore,

$$
\bar{\nabla} k_{n}(v)+\bar{\nabla} k_{n}\left(v_{1}\right)
$$

$$
\begin{gathered}
\left(y_{2}-x_{2}\right) \frac{\partial k_{n}}{\partial 1}\left(x_{1}, x_{2}, x_{3}\right)-\left(y_{1}-x_{1}\right) \frac{\partial k_{n}}{\partial 2}\left(x_{1}, x_{2}, x_{3}\right)-\left(y_{2}-x_{2}\right) \frac{\partial k_{n}}{\partial 1}\left(y_{1}, y_{2}, y_{3}\right) \\
+\left(y_{1}-x_{1}\right) \frac{\partial k_{n}}{\partial 2}\left(y_{1}, y_{2}, y_{3}\right)
\end{gathered}
$$

converges in $W^{-1,1}$. Moreover, the same formula holds if we change the indices 1,2 , and 3 by circular permutation. 
Taking the double partial derivative of this expression with respect to $x_{1}, y_{1}$, we obtain that

$$
-\frac{\partial^{2} k_{n}}{\partial 1 \partial 2}\left(x_{1}, x_{2}, x_{3}\right)-\frac{\partial^{2} k_{n}}{\partial 1 \partial 2}\left(y_{1}, y_{2}, y_{3}\right)
$$

converges in $W^{-3,1}$. Taking the double partial derivative of (14) with respect to $x_{1}$, $y_{2}$, we obtain that

$$
\frac{\partial^{2} k_{n}}{\partial 1^{2}}\left(x_{1}, x_{2}, x_{3}\right)-\frac{\partial^{2} k_{n}}{\partial 2^{2}}\left(y_{1}, y_{2}, y_{3}\right)
$$

converges in $W^{-3,1}$. Therefore,

$$
\frac{\partial^{2} k_{n}}{\partial 1 \partial 2}
$$

converges in $W^{-3,1}$ and there exists a sequence of real numbers $a_{n}$ such that

$$
\frac{\partial^{2} k_{n}}{\partial 1^{2}}+a_{n}
$$

converges in $W^{-3,1}$. Moreover, the same convergences holds with the same sequence $a_{n}$ when we change the indices 1,2 , and 3 by circular permutation.

Accordingly, there exist three sequences of real numbers $b_{n}^{1}, b_{n}^{2}, b_{n}^{3}$ such that

$$
\frac{\partial k_{n}}{\partial i}+a_{n} x_{i}+b_{n}^{i}
$$

converges in $W^{-2,1}$. Differentiating (15) with respect to $x_{1}$, we obtain that

$$
\begin{aligned}
& \left(y_{2}-x_{2}\right) \frac{\partial^{2} k_{n}}{\partial 1^{2}}\left(x_{1}, x_{2}, x_{3}\right)-\left(y_{1}-x_{1}\right) \frac{\partial^{2} k_{n}}{\partial 1 \partial 2}\left(x_{1}, x_{2}, x_{3}\right) \\
& +\frac{\partial k_{n}}{\partial 2}\left(x_{1}, x_{2}, x_{3}\right)-\frac{\partial k_{n}}{\partial 2}\left(y_{1}, y_{2}, y_{3}\right)
\end{aligned}
$$

converges in $W^{-2,1}$. Injecting $y_{2}=x_{2}$ in formula (17), Eq. (16) ensures that

$$
\frac{\partial^{2} k_{n}}{\partial 1 \partial 2}
$$

converges in $W^{-2,1}$. By the same argument, injecting $y_{1}=x_{1}$ in formula (17), Eq. (16) ensures that

converges in $W^{-2,1}$.

$$
\frac{\partial^{2} k_{n}}{\partial 1^{2}}+a_{n}
$$
that

Formulas (18) and (19) ensure the existence of a sequence $c_{n}$ of numbers such

$$
k_{n}(v)+\frac{1}{2} a_{n} v^{2}+b_{n} \cdot v+c_{n}
$$

converges weakly in $L^{1}\left(b_{n}\right.$ being the vector of components $\left.b_{n}^{i}\right)$. Finally, we obtain that $k_{n}$ converges weakly in $L^{1}(|v| \leqq R) / \Gamma$, which ensures that the image of $L$ is closed. Thus we can apply the open mapping theorem to $L$ in order to obtain a 
strictly positive $K_{R}$ such that

$$
\begin{aligned}
& \inf _{\text {functions } T} \int_{v^{2}+v_{1}^{2} \leqq R^{2}}\left|t(v)+t\left(v_{1}\right)-T\left(v+v_{1}, v^{2}+v_{1}^{2}\right)\right| d v d v_{1} \\
& \geqq K_{R} \inf _{m \in \Gamma} \int_{|v| \leqq R}|t(v)-m(v)| d v .
\end{aligned}
$$

Injecting $t=\log f$ in this estimate, we obtain Lemma 4.

The proof of Theorem 2 easily follows from Lemmas 3 and 4 together with estimate (13).

\section{On the Fokker-Planck-Landau Collision Kernel}

The derivation of the Fokker-Planck-Landau collision kernel may be found in [4 or 10]. We denote

$$
\begin{aligned}
Q^{\prime}(f, f)= & \operatorname{div}_{v} \int_{w \in \mathbb{R}^{3}}\left(\frac{C}{|v-w|}\right)\left\{I-\frac{(v-w) \otimes(v-w)}{|v-w|^{2}}\right\} \\
& \cdot\left\{f(w) \nabla_{v} f(v)-f(v) \nabla_{w} f(w)\right\} d w,
\end{aligned}
$$

where $C$ is a strictly positive constant depending on the physical properties of the gas, and $I$ is the identity tensor. We keep in this section the notations of Sects. 2 and 3. Moreover, we introduce $\Gamma^{\prime}$, the space of derivatives of logarithms of Maxwellians:

$$
\Gamma^{\prime}=\left\{a+b v / a \in \mathbb{R}^{3}, b \in \mathbb{R}\right\},
$$

and $H_{\log }^{1}$, the set of functions in $L^{2}$ such that their logarithm is in $H^{1}$.

The main result of this section is the following:

Theorem 3. Let $Q^{\prime}$ be the Fokker-Planck-Landau collision kernel, $R$ be strictly positive, and $\phi$ be as in Sect. 2. Then there exist a strictly positive $K_{R}$ such that for all $f$ in $L_{B}^{2} \cap H_{\log }^{1}$

$$
-\int_{v \in \mathbb{R}^{3}} Q(f, f)(v) \log f(v) d v \geqq C \frac{B_{R}^{2}}{2 R} K_{R} \inf _{m \in \Gamma^{\prime}} \int_{|v| \leqq R}\left|\nabla_{v} \log f(v)-m(v)\right|^{2} d v .
$$

Proof of Theorem 3. Boltzmann's $H$-theorem ensures that

$$
\begin{aligned}
- & \int_{v \in \mathbb{R}^{3}} Q^{\prime}(f, f)(v) \log f(v) d v \\
= & \frac{C}{2} \int_{v \in \mathbb{R}^{3}} \int_{w \in \mathbb{R}^{3}} f(v) f(w)\left\{\nabla_{v} \log f(v)-\nabla_{w} \log f(w)\right\}\left(\frac{1}{|v-w|}\right) \\
& \cdot\left\{I-\frac{(v-w) \otimes(v-w)}{|v-w|^{2}}\right\}\left\{\nabla_{v} \log f(v)-\nabla_{w} \log f(w)\right\} d w d v \\
\geqq & \frac{B_{R}^{2} C}{4 R} \int_{|v| \leqq R} \int_{|w| \leqq R}\left\{\nabla_{v} \log f(v)-\nabla_{w} \log f(w)\right\}\left\{I-\frac{(v-w) \otimes(v-w)}{|v-w|^{2}}\right\} \\
& \cdot\left\{\nabla_{v} \log f(v)-\nabla_{w} \log f(w)\right\} d w d v .
\end{aligned}
$$


But the eigenvalues of the symmetric tensor

$$
I-\frac{(v-w) \otimes(v-w)}{|v-w|^{2}}
$$

are 1 with order $n-1$ and 0 with order 1 . Moreover, the eigenvector corresponding to the eigenvalue 0 is $v-w$. Therefore, for all $x$ in $\mathbb{R}^{3}$,

$$
\left(I-\frac{(v-w) \otimes(v-w)}{|v-w|^{2}}\right) x \cdot x \geqq \inf _{\lambda \in \mathbb{R}}|x+\lambda(v-w)|^{2} .
$$

Accordingly, we obtain

$-\int_{v \in \mathbb{R}^{3}} Q(f, f)(v) \log f(v) d v$

$\geqq \frac{B_{R}^{2} C}{4 R} \quad \inf _{\text {functions } \lambda(v, w)} \int_{|v| \leqq R} \int_{|w| \leqq R}\left|\nabla_{v} \log f(v)-\nabla_{w} \log f(w)+\lambda(v, w)(v-w)\right|^{2} d w d v$.

Before going further in the proof, we need the following lemma:

Lemma 5. For all strictly positive $R$, there exists a strictly positive $K_{R}$ such that

$$
\begin{aligned}
& \quad \inf _{\text {functions } \lambda(v, w)} \int_{|v| \leqq R} \int_{|w| \leqq R}\left|\nabla_{v} \log f(v)-\nabla_{w} \log f(w)+\lambda(v, w)(v-w)\right|^{2} d w d v \\
& \geqq K_{R} \inf _{m \in \Gamma^{\prime}} \int_{|v| \leqq R}\left|\nabla_{v} \log f(v)-m(v)\right|^{2} d v .
\end{aligned}
$$

Proof of Lemma 5. Let $M^{\prime}$ be the space of functions of the form: $\lambda(v, w)(v-w)$, and let $L^{\prime}$ be the following operator:

$$
L^{\prime}: t \in L^{2}\left(|v| \leqq R ; \mathbb{R}^{3}\right) / \Gamma^{\prime} \rightarrow L^{\prime} t(v, w)=t(v)-t(w) \in L^{2}\left(|v| \leqq R,|w| \leqq R ; \mathbb{R}^{3}\right) / M^{\prime} .
$$

The operator $L^{\prime}$ is clearly linear and one-one (cf. [10]).

Observe that for all $t$ in $L^{2}\left(|v| \leqq R ; \mathbb{R}^{3}\right)$,

$$
\begin{aligned}
& \inf _{\text {functions } \lambda(v, w)} \int_{|v| \leqq R} \int_{|w| \leqq R}|t(v)-t(w)+\lambda(v, w)(v-w)|^{2} d w d v \\
& \leqq \inf _{a \in \mathbb{R}, b \in \mathbb{R}^{3}} \int_{|v| \leqq R} \int_{|w| \leqq R}|t(v)-t(w)+a(v-w)+b-b|^{2} d w d v \\
& \leqq 32 R^{3} \inf _{m \in \Gamma^{\prime}} \int_{|v| \leqq R}|t(v)-m(v)| d v .
\end{aligned}
$$

Therefore, the operator $L^{\prime}$ is continuous.

In order to apply the open mapping theorem, we have to prove that the image of $L^{\prime}$ is closed. Suppose that there exists a sequence $t_{n}$ in $L^{2}\left(|v| \leqq R ; \mathbb{R}^{3}\right) / \Gamma^{\prime}$ and $t$ in $L^{2}\left(|v| \leqq R,|w| \leqq R ; \mathbb{R}^{3}\right) / M^{\prime}$ such that $t_{n}(v)-t_{n}(w)$ tends to $t(v, w)$ in $L^{2}(|v| \leqq R$, $\left.|w| \leqq R ; \mathbb{R}^{3}\right) / M^{\prime}$. Then, there exists a sequence $k_{n}$ in $L^{2}\left(|v| \leqq R, \mathbb{R}^{3}\right)$, a sequence $\lambda_{n}$ of real-valued functions and $g$ in $L^{2}\left(|v| \leqq R,|w| \leqq R ; \mathbb{R}^{3}\right)$ such that $t_{n}$ is the natural projection of $k_{n}$ on $L^{2}\left(|v| \leqq R ; \mathbb{R}^{3}\right) / \Gamma^{\prime}, t$ is the natural projection of $g$ on 


$$
\begin{aligned}
& L^{2}\left(|v| \leqq R,|w| \leqq R ; \mathbb{R}^{3}\right) / M^{\prime}, \text { and } \\
& \qquad k_{n}(v)-k_{n}(w)+\lambda_{n}(v, w)(v-w) \rightarrow g(v, w)
\end{aligned}
$$

in $L^{2}\left(|v| \leqq R,|w| \leqq R, \mathbb{R}^{3}\right)$.

Accordingly, if we set $k_{n}=\left(k_{n}^{1}, k_{n}^{2}, k_{n}^{3}\right), v=\left(v_{1}, v_{2}, v_{3}\right), w=\left(w_{1}, w_{2}, w_{3}\right)$,

$$
\left(k_{n}^{i}(v)-k_{n}^{i}(w)\right)\left(v_{j}-w_{j}\right)-\left(k_{n}^{j}(v)-k_{n}^{j}(w)\right)\left(v_{i}-w_{i}\right)
$$

converges in $L^{2}\left(|v| \leqq R,|w| \leqq R ; \mathbb{R}^{3}\right)$ for all $i, j$ in $\{1,2,3\}$. Taking the double partial derivative of this expression with respect to $v_{i}, w_{i}$, we obtain that

$$
\frac{\partial k_{n}^{j}}{\partial i}(v)+\frac{\partial k_{n}^{j}}{\partial i}(w)
$$

converges in $H^{-2}$. Taking the double partial derivative of (22) with respect to $v_{i}, w_{j}$, where $i$ and $j$ are different, we obtain that

converges in $H^{-2}$. Therefore,

$$
-\frac{\partial k_{n}^{i}}{\partial i}(v)+\frac{\partial k_{n}^{j}}{\partial j}(w)
$$

$$
\frac{\partial k_{n}^{j}}{\partial i}
$$

converges in $H^{-2}$ for all distinct $i, j$ and there exists a sequence of real numbers $a_{n}$ such that

$$
\frac{\partial k_{n}^{i}}{\partial i}+a_{n}
$$

converges in $H^{-2}$. Accordingly, there exist three sequences of real numbers $b_{n}^{1}, b_{n}^{2}$, $b_{n}^{3}$ such that

$$
k_{n}^{i}+a_{n} v_{i}+b_{n}^{i}
$$

converges in $H^{-1}$. Differentiating (22) with respect to $v_{i}$, we obtain that

$$
\frac{\partial k_{n}^{i}}{\partial i}(v)\left(v_{j}-w_{j}\right)-\left(k_{n}^{j}(v)-k_{n}^{j}(w)\right)-\left(v_{i}-w_{i}\right) \frac{\partial k_{n}^{j}}{\partial i}(v)
$$

converges in $H^{-1}$. Injecting $v_{j}=w_{j}$ in formula (25), Eq. (23) ensures that:

$$
\frac{\partial k_{n}^{j}}{\partial i}
$$

converges in $H^{-1}$. In the same way, injecting $v_{i}=w_{i}$ in formula (25), Eq. (24) ensures that

$$
\frac{\partial k_{n}^{i}}{\partial i}+a_{n}
$$

converges in $H^{-1}$. Formulas (26) and (27) ensure that

$$
k_{n}^{i}+a_{n} v_{i}+b_{n}^{i}
$$

converges weakly in $L^{2}$. Finally, we obtain that $k_{n}$ converges weakly in $L^{2}(|v| \leqq R) / \Gamma^{\prime}$, which ensures that the image of $L^{\prime}$ is closed. Thus we can apply the 
open mapping theorem to $L^{\prime}$ in order to obtain a strictly positive $K_{R}$ such that

$$
\begin{aligned}
& \inf _{\text {functions } \lambda(v, w)} \int_{|v| \leqq R} \int_{|w| \leqq R}|t(v)-t(w)+\lambda(v, w)(v-w)|^{2} d w d v \\
& \geqq K_{R} \inf _{m \in \Gamma^{\prime}} \int_{|v| \leqq R}|t(v)-m(v)|^{2} d v .
\end{aligned}
$$

Injecting $t=\nabla_{v} \log f$ in this estimate, we obtain Lemma 5 .

The proof of Theorem 2 easily follows from Lemma 5 together with estimate (20).

\section{On the Linearised Kinetic Equations}

The proofs of the theorems of Sects. 2, 3 and 4 are still valid when we deal with the linearised collision terms of the Kač, Boltzmann or Fokker-Planck-Landau equations. More precisely, we obtain the following theorems, which are classical in the case of the Boltzmann and the Kač linearised equations:

We shall denote by $M$ a given Maxwellian, and $L^{2}\left(M^{1 / 2}(v) d v\right)$ the Hilbert space of functions $f$ such that $f M^{1 / 2}$ is in $L^{2}$, together with the norm:

$$
\|f\|_{L^{2}\left(M^{1 / 2}(v) d v\right)}=\left(\int f^{2}(v) M(v) d v\right)^{1 / 2} .
$$

Theorem 4. Denote by $\widetilde{Q}$ the Kač collision kernel. There exists a strictly positive $K$ such that for all $f$ in $L^{2}\left(M^{1 / 2}(v) d v\right)$ :

$$
-\int_{v \in \mathbb{R}} f(v) \tilde{Q}(M, M f)(v) d v \geqq K\left\|f-m_{f}\right\|_{L^{2}\left(M^{1 / 2}(v) d v\right)}^{2},
$$

where $m_{f}$ is the orthogonal projection of $f$ on $\tilde{\Gamma}$ in $L^{2}\left(M^{1 / 2}(v) d v\right)$.

Theorem 5. Denote by $Q$ the Boltzmann collision kernel with a cross-section $B$ such that

$$
B\left(v, v_{1}, \omega\right) \geqq C\left|\omega \cdot \frac{v_{1}-v}{\left|v_{1}-v\right|}\right|,
$$

where $C$ is a strictly positive constant. There exists a strictly positive $K$ such that for all $f$ in $L^{2}\left(M^{1 / 2}(v) d v\right)$ :

$$
-\int_{v \in \mathbb{R}^{3}} f(v) Q(M, M f)(v) d v \geqq K\left\|f-m_{f}\right\|_{L^{2}\left(M^{1 / 2}(v) d v\right)}^{2},
$$

where $m_{f}$ is the orthogonal projection of $f$ on $\Gamma$ in $L^{2}\left(M^{1 / 2}(v) d v\right)$.

Remark. The latter theorem is classical with additional assumptions on $B$, (cf. [3]). The author does not know whether the result given here in all its generality is new.

Theorem 6. Denote by $Q^{\prime}$ the Fokker-Planck-Landau collision kernel. There exists a strictly positive $K$ such that for all $f$ in $H^{1}$,

$$
-\int_{v \in \mathbb{R}^{3}} f(v) Q^{\prime}(M, M f)(v) d v \geqq K \inf _{m \in \Gamma^{\prime}} \int_{v \in \mathbb{R}^{3}} \zeta(v)|\nabla f(v)-\nabla m(v)|^{2} d v,
$$

where $\zeta$ is the function

$$
\zeta=\frac{1}{v} * M
$$




\section{On the Speed of Convergence to Equilibrium in Large Time}

Some results are already known on this subject. For example L. Arkeryd proved in [2] that the convergence to a Maxwellian in the case of the spatially homogeneous Boltzmann equation holds with exponential decay. However, it seems that for the full Boltzmann or Kač equations, the convergence is slower. This section is devoted to the explanation of how, in some sense, the convergence is in $O\left(\frac{1}{\sqrt{t}}\right)$. More
precisely, we are able to prove the following theorem:

Theorem 7. Let fbe a Di Perna-Lions solution of the Boltzmann equation (cf. [7]) with a cross section $B$ as in (12) and such that $f$ is in $L_{B}^{1}$. Then, for all strictly positive number $R$, there exists a strictly positive $K_{R}$ such that

$$
\int_{t}^{2 t} \int_{x \in \mathbb{R}^{3}} \inf _{m \in \Gamma} \int_{|v| \leqq R}|\log f-m| d v d x \frac{d t}{t}<\frac{K_{R}}{\sqrt{t}} .
$$

Proof of Theorem 7. We proceed as in Theorem 9 of [5]. According to [7],

$$
\int_{t=0}^{+\infty} \int_{x \in \mathbb{R}^{3}} \int_{v \in \mathbb{R}^{3}}-Q(f, f)(t, x, v) \log f(t, x, v) d v d x d t
$$

is finite and therefore

$$
\int_{t}^{2 t} \int_{x \in \mathbb{R}^{3}} \int_{v \in \mathbb{R}^{3}}-Q(f, f)(t, x, v) \log f(t, x, v) d v d x \frac{d t}{t} \leqq \frac{C}{t}
$$

for some constant $C$. According to Theorem 2, and using the fact that the function $\phi$ introduced in this theorem may be taken to be equivalent to $x^{2}$ when $x$ tends to 0 , we obtain that for some strictly positive $\mathrm{K}_{R}$ :

$$
\int_{t}^{2 t} \int_{x \in \mathbb{R}^{3}} \inf _{m \in \Gamma} \int_{|v| \leqq R}|\log f-m| \frac{d t}{t} d x d v<\frac{K_{R}}{\sqrt{t}} .
$$

Remark. The same kind of theorem would hold in the case of the Fokker-PlanckLandau equation if we knew the global existence of a weak solution.

\section{References}

1. Arkeryd, L.: On the long time behaviour of the Boltzmann equation in a periodic box (to appear)

2. Arkeryd, L.: Stability in $L^{1}$ for the spatially homogeneous Boltzmann equation. Arch. Rat. Mech. and Anal. 103, 151-167 (1988)

3. Cercignani, C.: The Boltzmann equation and its applications. Berlin, Heidelberg, New York: Springer 1988

4. Chapman, S., Cawlings, T.G.: The mathematical theory of non-uniform gases. London: Cambridge University Press 1939

5. Desvillettes, L.: Convergence to equilibrium in large time for Boltzmann and B.G.K. equations (to appear)

6. Desvillettes, L.: Une minoration du terme de dissipation d'entropie dans le modéle de Kač de la cinétique des gaz. C.R. Acad. Sci., Paris 307, 1955-1960 (1988) 
7. Di Perna, R., Lions, P.L.: On the Cauchy problem for Boltzmann equation, Global existence and weak stability. Ann. Math. (to appear)

8. Grad, H.: Principles of the kinetic theory of gases. In: Flügge's Handbuch der Physik 12, pp 205-294. Berlin, Göttingen, Heidelberg: Springer 1958

9. Kač, M.: Probability and related topics in the physical sciences. New York 1959

10. Lifschitz, E.M., Pitaevskii, L.P.: Physical kinetics. Oxford: Pergamon Press 1981

11. McKean, H.P.: Speed of approach to equilibrium for Kač's caricature of a Maxwellian gas. Arch. Rat. Mech. Anal. 21, 347-367 (1966)

12. Truesdell, C., Muncaster, R.: Fundamentals of Maxwell kinetic theory of a simple monoatomic gas. New York: Academic Press 1980

Communicated by J.L. Lebowitz

Received February 10, 1989 